\title{
Quantum Rabi Oscillation: A Direct Test of Field Quantization in a Cavity
}

\author{
M. Brune, F. Schmidt-Kaler, A. Maali, J. Dreyer, E. Hagley, J. M. Raimond, and S. Haroche \\ Laboratoire Kastler Brossel,* Département de Physique de l'Ecole Normale Supérieure, 24 rue Lhomond, \\ F-75231 Paris Cedex 05, France
}

(Received 9 November 1995)

\begin{abstract}
We have observed the Rabi oscillation of circular Rydberg atoms in the vacuum and in small coherent fields stored in a high $Q$ cavity. The signal exhibits discrete Fourier components at frequencies proportional to the square root of successive integers. This provides direct evidence of field quantization in the cavity. The weights of the Fourier components yield the photon number distribution in the field. This investigation of the excited levels of the atom-cavity system reveals nonlinear quantum features at extremely low field strengths.
\end{abstract}

PACS numbers: 42.50.Ar, 03.65.-w, 32.80.-t

Since Planck's hypothesis, the quantization of radiation is a universally accepted fact of nature. Besides the blackbody radiation law, many phenomena such as the Compton effect, spontaneous emission, and radiative QED corrections point to the existence of field quanta. In quantum optics, nonclassical field behaviors, such as squeezing, antibunching, or sub-Poissonian noise statistics have been recently demonstrated [1]. However, the most generally admitted evidence of field quantization, the discrete nature of the photodetection current, is perfectly explained by a classical description of the field, provided that the linear detector is a quantum system [2]. Another simple fact, granted in all quantum field descriptions, i.e., the discreteness of the energy of the radiation stored in a cavity mode, has up to now escaped direct observation. Obviously, a detector more subtle than an ordinary linear photodetector counting "clinks" is required. Other difficulties also conspire against field quantization evidence. When the field energy is large compared to the quantum, incremental photon number changes are unnoticeable. Quite generally, cavity relaxation tends to blurr the photon number and to make field measurements sensitive to average values only, which behave as classical variables.

The study of the Jaynes-Cummings Hamiltonian [3], which describes the ideal coupling of a two-level atom to a single field mode, indicates that a signature of the discrete nature of field quanta could be provided by the observation of a single atom's Rabi nutation in a weak radiation field. This effect corresponds to the population oscillations between two atomic levels $e$ and $g$, when the field is resonant on the $e \rightarrow g$ transition. Usually, the field presents a dispersion of photon numbers. If it is thermal, the probability $P(n)$ of finding $n$ photons is exponential, while it is Poissonian for a coherent field. When relaxation is negligible, the Rabi oscillation is predicted to be a superposition of sinusoidal terms, each corresponding to an $n$ value. The weights of the various components in the sum reflect the $P(n)$ distribution.

For an atom initially in the upper state $e$, the probability $P_{e g}(t)$ to find it at a later time $t$ in $g$ is $P_{e g}(t)=$ $\Sigma_{n} P(n) \sin ^{2} \Omega \sqrt{n+1} t$, where $\Omega$ is the intrinsic atom-field coupling and the $\sqrt{n+1}$ terms represent, for each photon number $n$, the dimensionless field amplitude relevant for an atomic emission process [4]. For large coherent fields, the relative dispersion of $n$ values is negligible and, during realistic observation times, the Rabi nutation practically occurs at a single angular frequency $2 \Omega \sqrt{\bar{n}+1}$, associated with the mean photon number $\bar{n}$ (classical limit). Quantum behavior of the Rabi nutation can be observed only when the coherent field is weak, and the $n$ fluctuations relatively important. The beating between the uncommensurate frequencies is then expected to produce a collapse of the oscillation amplitude, followed at a later time by a revival [5].

An experiment on the Rydberg atom micromaser [6] has revealed an oscillation of the atomic population in a thermal field $(1.5<\bar{n}<3.8)$ and in the micromaser field. The limited range of interaction times did not provide enough resolution to separate frequencies associated with successive $n$ values. The signal in the micromaser field exhibited features similar to the "collapse and revival" effect expected in a coherent field. However, the atom was not only the probe but also the source of the field, whose statistics were changing with interaction time.

We describe here the observation of the Rabi nutation in the vacuum and in a weak coherent field. Atomic emission effects have negligible influence. The atomcavity interaction time and the cavity damping time are long enough to permit the resolution of discrete frequencies proportional to the sequence of successive square root integers. This experiment provides a direct evidence of field energy quantization in a cavity mode.

The setup, sketched in Fig. 1, is cooled to $0.8 \mathrm{~K}$. Rubidium atoms, effusing from the oven $O$, are prepared by a time resolved process into the circular Rydberg state $e$ (principal quantum number 51) in the box $B$ [7]. At a repetition rate of $660 \mathrm{~Hz}, 2 \mu$ s long pulses of Rydberg atoms start from $B$ with a Maxwellian velocity spread (mean velocity $v_{0}=350 \mathrm{~m} / \mathrm{s}$ ). The atoms cross the cavity $C$ made of two niobium superconducting mirrors (diameter $5 \mathrm{~cm}$, radius of curvature $4 \mathrm{~cm}$, mirror separation $2.75 \mathrm{~cm}$ ). This cavity, whose axis is vertical, sustains the two TEM $_{900}$ 


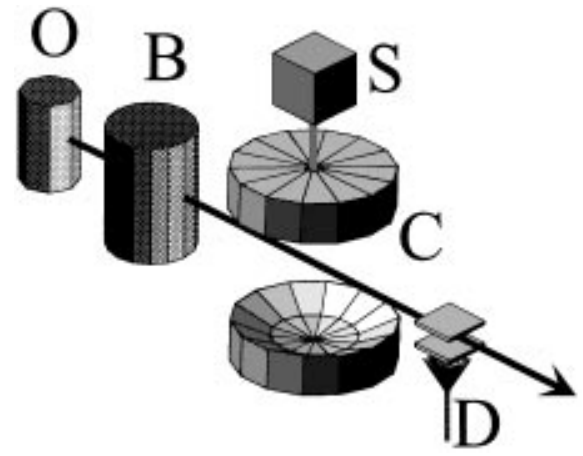

FIG. 1. Sketch of the experimental setup.

modes with orthogonal linear polarizations and transverse Gaussian profiles (waist at center $w=5.96 \mathrm{~mm}$ ). Because of a slight mirror ellipticity, the mode degeneracy is lifted (splitting $111 \mathrm{kHz}$ ). The lower frequency mode is tuned into resonance with the $e$ to $g$ transition between adjacent circular Rydberg states with principal quantum numbers 51 and 50 (frequency $51.099 \mathrm{GHz}$ ). A small static electric field $(0.36 \mathrm{~V} / \mathrm{cm})$ is applied across the mirrors to stabilize the circular state orbit in the horizontal plane and to provide fine tuning of the atomic frequency (via the Stark effect). This field can also be set to a larger value to detune the atom and the cavity by an amount $(1 \mathrm{MHz})$ which makes the interaction between them negligible. The mode $Q$ factor is $7 \times 10^{7}$, corresponding to a photon lifetime $T_{\text {cav }}=220 \mu \mathrm{s}$, which is longer than the atom-cavity interaction time. A very stable source $S$ is used to inject continuously into the cavity a small coherent field with a controlled energy varying from zero to a few photons. The atoms are detected after the cavity by state selective field ionization (detector $D$ ) and the transfer rate from $e$ to $g$ is measured.

In circular Rydberg atoms [8], the valence electron is confined near the classical Bohr orbit. These atoms have a long radiative lifetime (32 and $30 \mathrm{~ms}$ for $e$ and $g$, respectively), which makes atomic relaxation negligible during the atom transit time across the apparatus. These atoms are strongly coupled to radiation and the atomfield coupling at cavity center, $\Omega_{0} / 2 \pi=25 \mathrm{kHz}$, is entirely determined by the size of the Bohr orbit and the volume of the cavity mode [7]. In fact, the coupling varies along the atom trajectory according to the law $\Omega(z)=\Omega_{0} \exp \left(-z^{2} / w^{2}\right)$, where $z$ is the position of the atom along the beam axis $(z=0$ at cavity center). The atomic beam, which has a vertical dispersion of $0.5 \mathrm{~mm}$, is adjusted to cross the cavity at an antinode level.

It is important to keep the atomic flux low enough to avoid field buildup by cumulative atomic emission (micromaser effect [6]). The average delay between successive atoms is adjusted to be $2.5 \mathrm{~ms}$, much longer than $T_{\text {cav }}$. Each atom thus experiences a field restored by $S$ to its initial state. Taking into account the detection efficiency, the actual counting rate is $30 \mathrm{~s}^{-1}$.

The control of the atom-cavity interaction time $t$ is essential. First, we determine to an accuracy of $1 \%$ the velocity $v$ of each detected atom from the knowledge of its arrival time in $D$ and of its preparation time in $B$. We then deduce an effective interaction time $t$ by the relation $t=\left(1 / \Omega_{0}\right) \int_{-\infty}^{\infty} \Omega(z) d z / v=\sqrt{\pi} w / v$. The Maxwell velocity distribution yields reasonable atomic statistics in the range $250<v<700 \mathrm{~m} / \mathrm{s}$, i.e., $15<t<$ $40 \mu \mathrm{s}$. This corresponds to the central part of the $P_{e g}(t)$ signal, recorded in about $40 \mathrm{~min}$. For longer times, we select slower atoms, which forces us to increase the overall atomic flux. To avoid cavity field buildup due to emission by fast atoms, we apply on the cavity mirrors a pulse of detuning field which is switched off just before the slow atoms enter the cavity. In this way, we reach $v$ values in the range 110 to $250 \mathrm{~m} / \mathrm{s}$, corresponding to $40<t<$ $90 \mu \mathrm{s}$. Recording this part of the signal takes $1 \mathrm{~h}$. Finally, we proceed to record the signal corresponding to short interaction times ( 0 to $15 \mu \mathrm{s}$ ). We detect fast atoms and we further reduce $t$ with the help of the detuning electric field. In each sequence this field is switched on at a preset time $t_{1}$, corresponding to an atomic position $z_{1}\left(v, t_{1}\right)$ inside the cavity. The interaction time $t$ is then shortened to the value $t=\left(1 / \Omega_{0}\right) \int_{-\infty}^{z_{1}\left(v, t_{1}\right)} \Omega(z) d z / v$. This part is recorded in $40 \mathrm{~min}$. The three parts are then combined and we check that they merge smoothly. Each recording corresponds to about $2 \times 10^{5}$ detected atoms.

The signals are presented in Fig. 2. Figures 2(A) to 2(D) show the Rabi nutations for increasing field amplitudes. Figure 2(A) presents the nutation in cavity vacuum (with a very small correction due to thermal field effects). Four oscillations are observed, up to $2 \Omega_{0} t=$ $8 \pi$. This signal exhibits the reversible spontaneous emission and reabsorption of a single photon in an initially empty cavity mode, an effect predicted by the Jaynes-Cummings model but never observed so far in the time domain. When a small coherent field is injected [Figs. 2(B), 2(C), and 2(D)], the signal is no longer sinusoidal, as it would be for an atom interacting with a classical field. In Figs. 2(C) and 2(D), after a first oscillation, a clear collapse and revival feature is observed [5]. Cavity relaxation plays a marginal role in the decrease of the oscillation amplitude in the $0-100 \mu \mathrm{s}$ time range (it would lead to complete transfer from $e$ to $g$ at times much longer than $220 \mu \mathrm{s}$ ). Dark counts in the ionization detectors are one of the main causes of oscillation damping (they become increasingly important at long times, i.e., low atomic fluxes). Decoherence by collisions with background gas may also contribute to the oscillation relaxation.

Figures 2(a) to 2(d) show the Fourier transform of the nutation signal, obtained after symmetrization with respect to $t=0$. Discrete peaks at frequencies $\nu=$ $47 \mathrm{kHz}, \nu \sqrt{2}, \nu \sqrt{3}$, and even $2 \nu$ are clearly observable, revealing directly the quantized nature of the field up to three photons. The frequency $\nu$ is in good agreement with the expected value $\Omega_{0} / \pi=50 \mathrm{kHz}$. The low frequency noise in these spectra is an artifact due to a slow modulation in the signal to noise ratio introduced by 
our data collection procedure. Note also the scale change from Fig. 2(a) to 2(d). We have checked that the total area of the Fourier transform curve remains constant, as required by $P(n)$ normalization. The height of the Fourier peaks thus decreases with the field amplitude, explaining the decrease in the signal to noise ratio from 2(a) to 2(d).

The time dependent signals are fitted by a sum of damped sinusoids, with frequencies $\nu \sqrt{n+1}, n$ varying from 0 to 5 [solid lines in Figs. 2(A) to 2(D)]. The agreement is very good. From the relative weights of the terms in these fits, we determine photon number probabilities, shown in Figs. $2(\alpha),(\beta),(\gamma)$, and $(\delta)$. When no field is injected [Fig. $2(\alpha)$ ], this distribution fits the thermal radiation law (solid line) with the very small average photon number $\bar{n}=0.06( \pm 0.01)$, corresponding well with the value deduced from the cavity temperature $(0.05$ photon at $T=0.8 \mathrm{~K})$. With an injected coherent field [Figs. 2( $\beta)$ to $2(\delta)$ ], there is a very good agreement between the experimental data and a Poisson law (solid lines), providing an accurate value of the mean photon number in each case: $0.40( \pm 0.02), 0.85( \pm 0.04)$, and $1.77( \pm 0.15)$, respectively. The residual thermal field causes no appreciable deviation from the Poisson law for these mean photon numbers.

This experiment can also be viewed as a measurement of the atom-cavity spectrum [9], deduced from the JaynesCummings Hamiltonian [3]. The excited levels of this system are organized in doublets, separated by one field
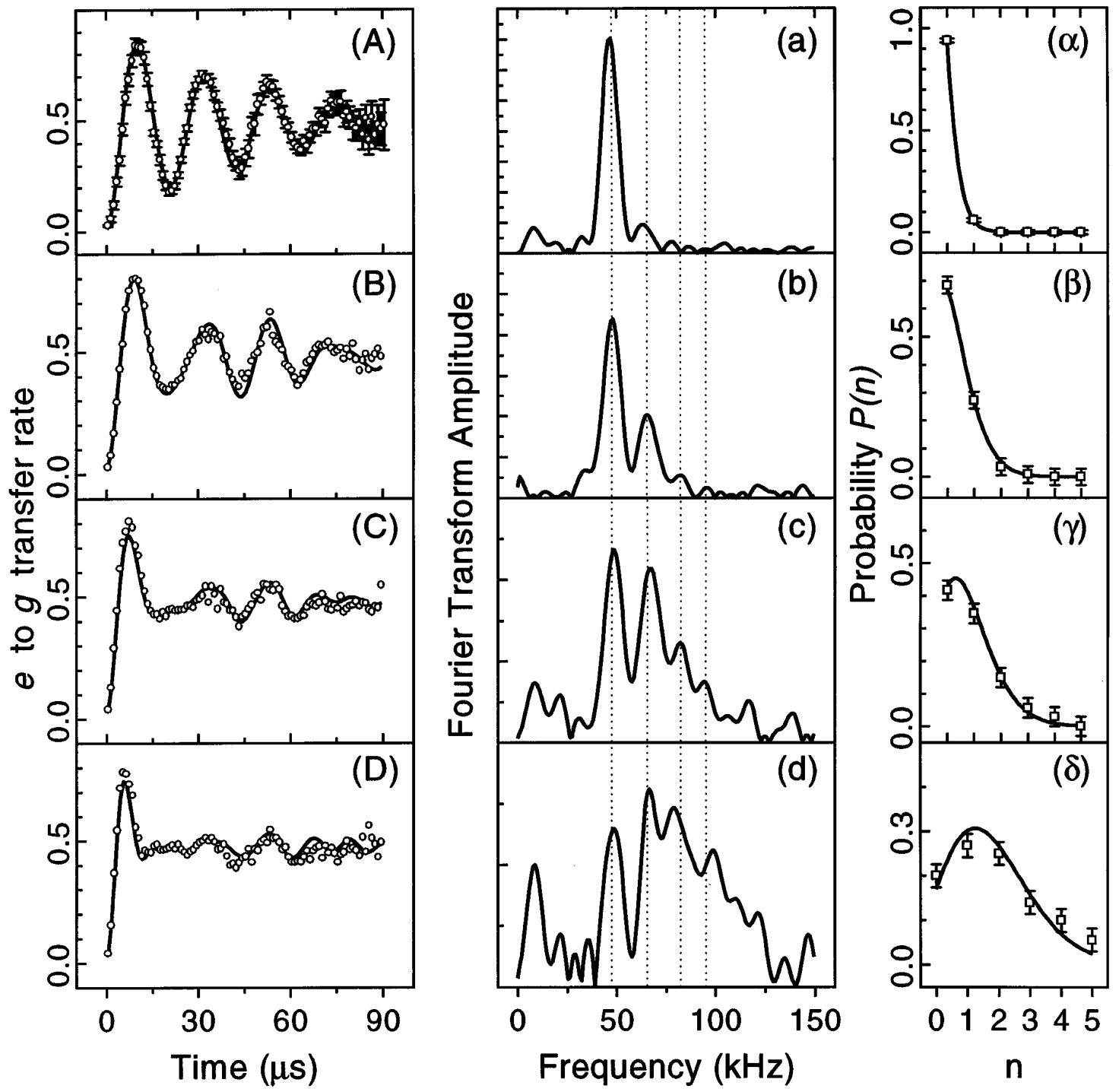

FIG. 2. (A), (B), (C), and (D): Rabi nutation signal representing $P_{e, g}(t)$, for fields with increasing amplitudes. (A) No injected field and $0.06( \pm 0.01)$ thermal photon on average; (B), (C), and (D) coherent fields with $0.40( \pm 0.02), 0.85( \pm 0.04)$, and $1.77( \pm 0.15)$ photons on average. The points are experimental [errors bars in (A) only for clarity]; the solid lines correspond to theoretical fits (see text). (a), (b), (c), (d) Corresponding Fourier transforms. Frequencies $\nu=47 \mathrm{kHz}, \nu \sqrt{2}, \nu \sqrt{3}$, and $2 \nu$ are indicated by vertical dotted lines. Vertical scales are proportional to $4,3,1.5$, and 1 from (a) to $(\mathrm{d})$. $(\alpha),(\beta),(\gamma),(\delta)$ Corresponding photon number distribution inferred from experimental signals (points). Solid lines show the theoretical thermal $(\alpha)$ or coherent $[(\beta),(\gamma)$, $(\delta)]$ distributions which best fit the data. 
quantum. The splittings of doublets corresponding to increasing energies are precisely $h \nu, h \nu \sqrt{2}, h \nu \sqrt{3}, \ldots$ The Rabi nutation is thus a quantum beat signal, resulting from the coherent excitation and detection of linear superpositions of all these levels. The spectral component at frequency $\nu$, the only one to be excited if the field is in the vacuum state, reveals the splitting of the first manifold, already observed in direct spectroscopic investigations (vacuum Rabi splitting) [10]. The other components are associated with more excited manifolds, which are resolved in this work for the first time.

The first component of the spectrum (frequency $\nu$ ) can be explained by a linear coupled oscillator model of the atom-cavity system [11]. The increase by discrete steps of the atom-field coupling revealed by the existence of the other components is a quantum nonlinear effect directly related to the saturation of the two level atom resonance. Nonlinear effects are observed here with less than half a photon (energy smaller than $10^{-4} \mathrm{eV}$ ). The nonlinearity of the atom-field coupling at very low field strength makes the Rydberg atom very different from an ordinary photodetector. It is the essential feature which renders the atom dynamics sensitive to the quantum behavior of the field.

This resonant experiment dramatically shows once more that circular Rydberg atoms are very sensitive probes of millimeter wave fields, able to measure not only the mean field intensity with subphoton sensitivity, but also to determine accurately its statistics. Dispersive field detection methods, also using circular Rydberg atoms, have already demonstrated comparable sensitivity with the added potential of being quantum nondemolition [7]. The combination of resonant and dispersive methods using Rydberg atoms and microwave cavities opens the way to many fascinating applications for the measurement and manipulation of weak quantum fields and for quantum information processing [12].

We would like to thank B. Bonin and H. Safa (CEA Saclay) for helping us in the preparation of the superconducting cavity. One of us (F.S. K.) has been supported by an individual EEC grant.

*Laboratoire de l'Université Pierre et Marie Curie et de l'ENS, associé au CNRS (URA18).

[1] D. F. Walls and G. J. Milburn, Quantum Optics (SpringerVerlag, New York, 1995).

[2] W.E. Lamb and M. O. Scully, in Polarisation, Matière et Rayonnement (PUF, Paris, 1968), p. 363; M. O. Scully and M. Sargent III, Phys. Today 25, No. 3, 38 (1972); L. Mandel, Prog. Opt. XIII, 27 (1976).

[3] E. T. Jaynes and F.W. Cummings, Proc. IEEE 51, 89 (1963).

[4] If we considered instead a Rabi oscillation in absorption (atom initially in $g$ ), $\sqrt{n}$ would replace $\sqrt{n+1}$ in the expression of the atomic transition probability.

[5] J.H. Eberly, N.B. Narozhny, and J.J. SanchezMondragon, Phys. Rev. Lett. 44, 1323 (1980).

[6] G. Rempe, H. Walther, and N. Klein, Phys. Rev. Lett. 58, 353 (1987).

[7] M. Brune, P. Nussenzveig, F. Schmidt-Kaler, F. Bernardot, A. Maali, J. M. Raimond, and S. Haroche, Phys. Rev. Lett. 72, 3339 (1994).

[8] R. G. Hulet and D. Kleppner, Phys. Rev. Lett. 51, 1430 (1983).

[9] P. Alsing, D. S. Guo, and H. J. Carmichael, Phys. Rev. A 45, 5135 (1992).

[10] R. J. Thompson, G. Rempe, and H. J. Kimble, Phys. Rev. Lett. 68, 1132 (1992); F. Bernardot, P. Nussenzveig, M. Brune, J. M. Raimond, and S. Haroche, Europhys. Lett. 17, 33 (1992).

[11] Y. Zhu, D. J. Gauthier, S.E. Morin, Q. Wu, H.J. Carmichael, and T.W. Mossberg, Phys. Rev. Lett. 64, 2499 (1990).

[12] A. Barenco, D. Deutsch, A. Ekert, and R. Josza, Phys. Rev. Lett. 74, 4083 (1995); T. Sleator and H. Weinfurter, Phys. Rev. Lett. 74, 4087 (1995); P. Domokos, M. Brune, J. M. Raimond, and S. Haroche, Phys. Rev. A 52, 3554 (1995). 